\title{
Deciphering the tissue-specific regulatory role of intronless genes across cancers
}

\author{
Katia Aviña-Padilla', José Antonio Ramírez-Rafael', Gabriel Emilio Herrera-Oropeza², Guillermo Romero², \\ Octavio Zambada-Moreno', Ishaan Gupta ${ }^{4}$, and Maribel Hernández-Rosales ${ }^{*}$
}

'CINVESTAV-Irapuato; 'King's College London; ${ }^{3}$ Data-Pop Alliance NY; ${ }^{4}$ Indian Institute of Technology, Delhi.

"maribel.hr@cinvestav.mx

\begin{abstract}
Intronless genes (IGs) or single-exon genes lacking an intron are found across most Eukaryotes. Notably, IGs display a higher transcriptional fidelity as they are not regulated through alternative splicing, suggesting better predictability biomarkers and easier regulation as targets for therapy. Cancer is a complex disease that relies on progressive uncontrolled cell division linked with multiple dysfunctional biological processes. Tumor heterogeneity remains the most challenging feature in cancer diagnosis and treatment. Given the clinical relevance of IGs, we aim to identify their unique expression profiles and interactome, that may act as functional signatures across eight different cancers. We identified 940 protein-coding IGs in the human genome, of which about $35 \%$ were differentially expressed across the analyzed cancer datasets. Specifically, $\sim 78 \%$ of differentially expressed IGs were undergoing transcriptional reprogramming with elevated expression in tumor cells. Remarkably, in all the studied tumors, a highly conserved induction of a group of deacetylase-histones located in a region of chromosome 6 enriched in nucleosome and chromatin condensation processes. This study highlights that differentially expressed human intronless genes across cancer types are prevalent in epigenetic regulatory roles participating in specific PPI networks for ESCA, GBM, and LUAD tumors. We determine that IGs play a key role in the tumor phenotype at transcriptional and post-transcriptional levels, with important mechanisms such as interactomics rewiring.
\end{abstract}

Keywords: single-exon genes, comparative genomics, cancer genomics, epigenetics, tumor heterogeneity, cancer evolutionary genomics.

\section{Introduction}

Most eukaryotic gene structures contain exons interrupted by non-coding introns that are removed by RNA splicing to generate the mature mRNA. Although the most prevalent class of genes in the human genome contain Multiple exon genes (MEGs), about 5\% of the genes are intronless (IGs) or single-exon (SEGs) that lack introns. Due to the absence of introns and associated post-transcriptional splicing, IGs entail a higher transcriptional fidelity suggesting a potential role as clinical biomarkers and drug targets that deserve careful consideration in diseases such as cancer [1-3]. Several previous studies have identified the role of IGs in cancer [2, 4-7]. For example the $R P R M$ gene increased cell proliferation and tumor suppression activity in gastric cancer [7]; CLDN8 gene is associated with colorectal carcinoma and renal cell tumors [6]; while ARLTS1 is upregulated in melanoma; PURA \& TAL2 are upregulated in leukemia [2] and protein kinase $C K 2 \alpha$ gene is up-regulated in all human cancers [4]; 
A remarkable instance of IGs acting in a clinical role is $S O X 11$, a member of SOXC (SRY-related HMG-box) gene family of transcription factors involved in embryonic development and tissue remodeling by participating in cell fate determination [8]. SOX11 has been associated with tumorigenesis, with aberrant nuclear protein expression in Mantle Cell Lymphoma (MCL) patients [9-12]. This TF is not expressed in normal lymphoid cells or other mature B cell lymphomas (except Burkitt lymphoma), but it is highly expressed in conventional MCL, including the cyclin D1-MCL subtype [5]. Hence, SOX11 represents a widely used marker in the differential diagnosis of MCL and other types of small B-cell neoplasias in the clinical hemato-oncology practice [13-14].

However, to date, a comparative analysis integrating transcriptomic and interactomics profiles of IGs across diverse types of cancer is missing. Hence, this work aims to identify and characterize their expression, functional role, and interactomics profiles across different selected cancers.

\section{Results}

In this study we use RNA-sequencing data from 3880 tumor samples belonging to 8 cancer types from the cancer genome atlas or TCGA (Supplementary Table 1). We selected the four most prevalent cancers, namely Breast Invasive Carcinoma (BRCA), Colon Adenocarcinoma (COAD), Lung Adenocarcinoma (LUAD), Prostate Adenocarcinoma (PRAD); along with the four most aggressive cancers with high intrinsic heterogeneity, namely Bladder Urothelial Carcinoma (BLCA), Esophageal Carcinoma (ESCA), Glioblastoma Multiforme (GBM), and Kidney Renal Clear Cell Carcinoma (KIRC). We found that 338 out of the 940 genes identified as IG-encoded proteins are undergoing differential regulation in tumors compared to the normal tissue. GBM had the most number of DE-IGs at 168, followed by KIRC at 130, and both PRAD and COAD at 116. While in BRCA 104, LUAD 87, BLCA 97 and ESCA 86 were determined.

\subsection{IGs tend to have a more induced gene expression pattern when compared to MEGs}

In order to characterize the distinct biological behavior of IGs, we studied the overall gene expression patterns of IGs compared to those of the multi-exonic genes (MEGs) in all the tumors. Notably, a greater percentage of upregulated genes are found among DE-IGs than DE-MEGs in all the different types of cancers analyzed.

For instance, when comparing both populations of genes, we identified statistical significance for an over-representation of the up-regulated IGs over the MEGs group among DEGs in PRAD $(p$-value $=2.8108$ e-07); ESCA ( $p$-value $=7.0858$ e-05); LUAD ( $p$-value $=0.0015)$, and BRCA $(p$-value $=0.0276)$ cancers. Moreover, we aimed to compare if the upregulation levels in means of expression ranges are higher in IGs than in MEGs transcripts. Our analysis revealed that in almost all the cancers under study, except in lung adenocarcinoma, IGs tend to express in higher ranges than MEGs, as shown in Supplementary Figure 1. Further, we found a high proportion of IGs encodes for histone proteins involved in chromatin structure and other proteins essential to the regulation of development, growth, and proliferation (Supplemental Figure 2). 


\subsection{Upregulated IGs across cancer types encode for highly conserved HDAC deacetylate histones involved in negative gene regulation}

To dig insight into the role of the prevalent upregulation mechanism identified for the DE-IGs, we aimed to characterize the groups of induced IGs across the analyzed cancer types. 338 out of $940(\sim 35 \%)$ IG encoded proteins in the human genome, are differentially expressed across the eight analyzed tumor types. In a high number of them, $222(\sim 78 \%)$, upregulation is conserved in two or more cancers, which suggests they are undergoing transcriptional reprogramming with higher rates of upregulated levels of their mRNAs as an outcome. Moreover, this upregulation mechanism is highly shared among the different cancers (Figure 1). Most of the genes upregulated in BLCA and ESCA are shared with the other tumors, while tumors with a significant but less shared upregulation are GBM, PRAD, and KIRC, which could be expected given their remarkable heterogeneity (Figure 1a).
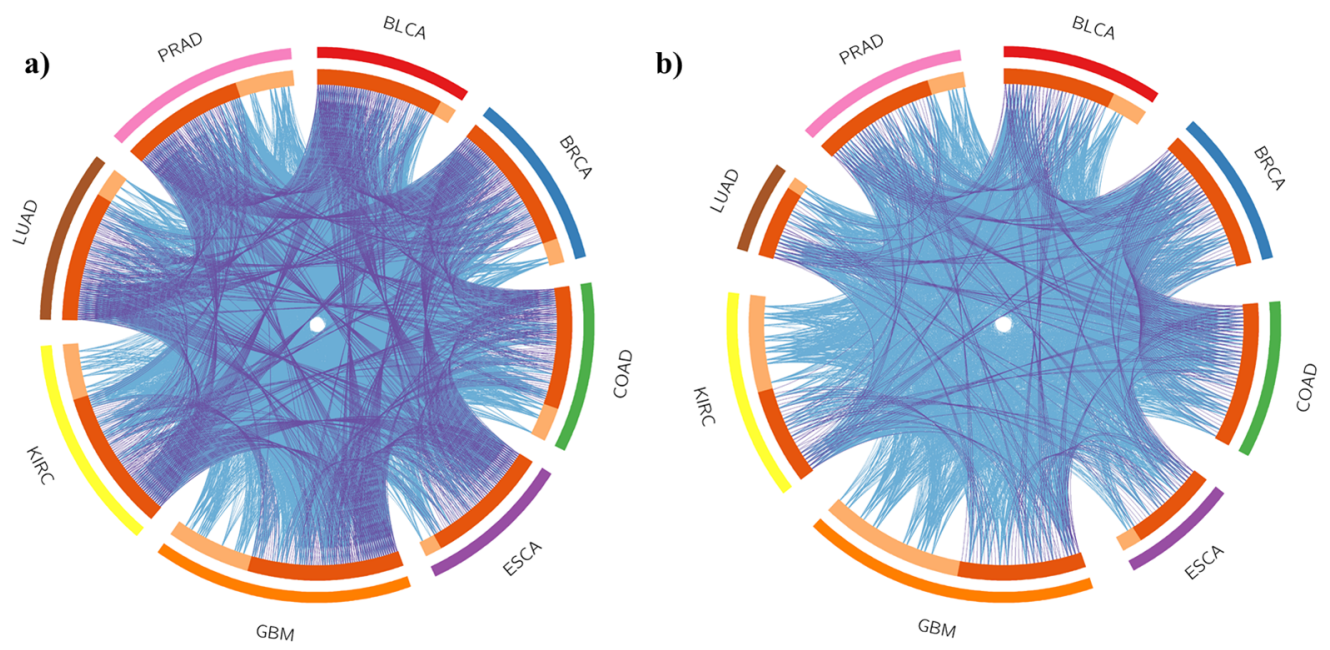

Figure 1. Deregulated IGs across the analyzed cancer genomes. The Circos plot depicts how deregulated genes for each cancer overlap. On the outside shell, each arc represents a cancer genome: BLCA in red, BRCA in blue, COAD in green, ESCA in purple, GBM in orange, KIRC in yellow, LUAD in brown, and PRAD in pink. On the inside shell, the dark orange color represents the proportion of DE genes shared with other cancers, while the light orange color represents the proportion of genes that are uniquely deregulated in a cancer. Purple arcs represent shared DE genes, while blue arcs represent shared functional enrichment terms among cancers. The greater the number of purple arcs and the longer the dark orange area imply a greater overlap among the deregulated IGs across cancers. (a) shows the upregulated genes, while (b) the downregulated.

To delve insights into the conservation of the activation of this negative gene expression mechanism in the cancer genomes, we identify the most conserved upregulated histones in all the diseases. We identified a group of 13 histone-related genes whose expression is highly conserved among the cancers (Supplementary Figure 3). Moreover, this group of upregulated genes is statistically enriched in a proximal region (26.1968-27.8927 position in Mbps ) in chromosome 6 in the human genome (Supplementary Figure 4). 
Haspin was found to be the only DE-IG shared among all the tumor genomes, highlighting its relevance in tumorigenesis. This gene is known to be required for histone H3 phosphorylation, necessary for chromosomal passenger complex accumulation at centromeres of mitotic cells [15-16]. In addition to its chromosomal association, it is associated with centrosomes and spindles during mitosis. The overexpression of this gene is related to a delayed progression through early mitosis [15].

Functional enrichment analysis was carried out for the deregulated IGs in all the studied diseases (Supplementary Table 2). GBM is the disease with the most DE-IGs and with the most diverse functional roles for the induced genes. The GBM-specific enriched terms are pathways of neurodegeneration, cell-cell adhesion, and gland development. The cancer-specific enriched terms chaperone-mediated protein folding, and regulation of neuron apoptotic processes were found for esophagus and colon cancers, respectively. Further, the Reactome pathways $R$-HSA-321481 deacetylases histones (HDACs), and $R$-HSA-3214858 RMTs methylate histone arginines were also enriched in IGs while GO:0006335 term: DNA replication-dependent chromatin assembly was also enriched suggesting an essential role in cancer biology.

\subsection{IG downregulation is conserved in breast and colon cancers and is involved in signaling and cell-specific functions.}

When comparing the downregulated IGs and their enriched functional terms among the diseases, it was pointed out that the breast and colon cancers share all the repressed IGs with their functional pattern. Downregulated genes in those cancers are involved in the regulation of cellular localization, sensory organ development, regulation of membrane potential, adipogenesis, epithelial to mesenchymal transition in colorectal cancer, growth, actin filament base process, positive regulation of cell death, class A/1 (rhodopsin-like receptors), and the regulation of secretion by cell biological processes, (Supplementary Table 2). Notably, the most shared downregulated pathway among cancer genomes is the regulation of anatomical structural size, shared among BRCA, PRAD, GBM, LUAD, and BLCA tumors. Additionally, unique roles are found in lung and prostate cancers. They have downregulated genes involved in the vitamin $\mathrm{D}$ receptor pathway, and wounding response respectively.

\subsection{Cancer-specific differentially expressed IGs}

To study the specificity in the regulation of human IGs, we quantified the number of cancer-specific DE-IGs (Figure 2). We also built a bipartite network to identify cancer-specific and shared deregulated IGs, where upper nodes represent DE-IGs, and bottom nodes represent cancer types, we link an IG to a cancer if it was found to be deregulated in that cancer (Supplementary Figure 2). Among the 338 DE-IGs, we identified that $\sim 35 \%$ were specific for a cancer type (Supplementary Table 3). GBM followed by KIRC and PRAD with 35, 18, and 16 DE-IGs respectively displayed the highest specificity for IG expression. 


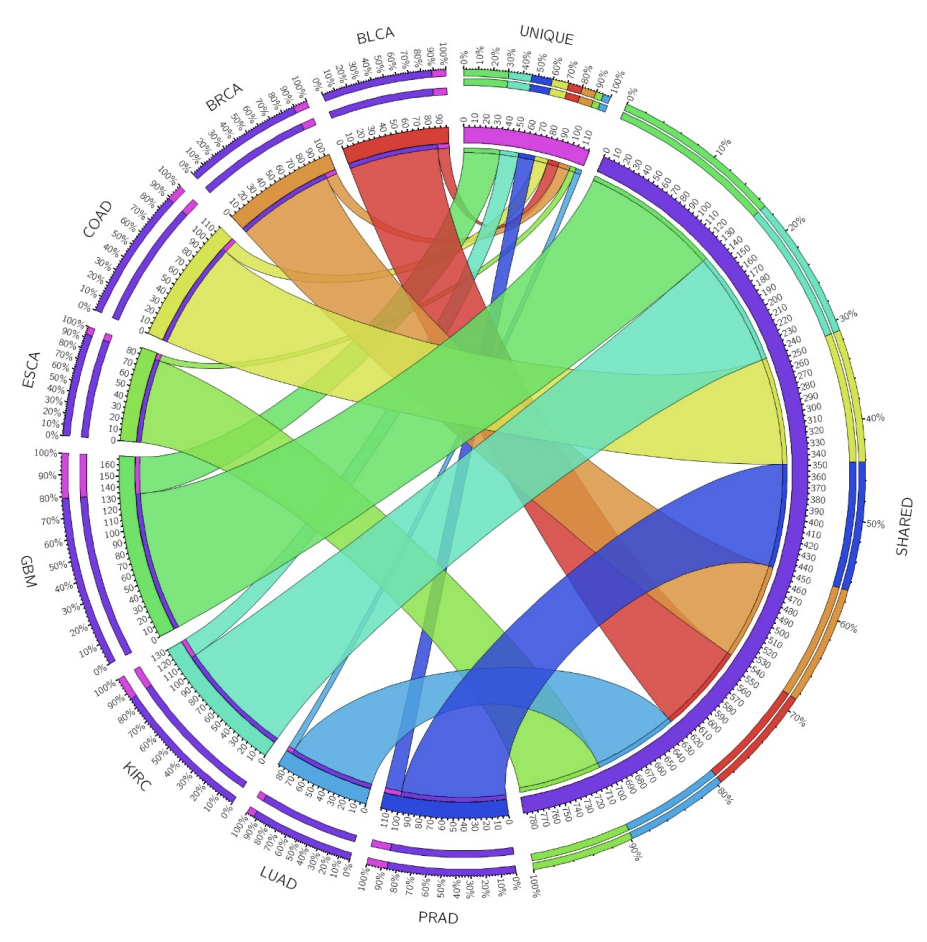

Figure 2. Shared and unique differentially expressed IGs across cancers. Circa plot depicts the number of shared DE-IGs across the 8 types of tumors under study. In the left hemisphere, the total number of DE-IGs in each cancer is represented by an arc: BLCA (red), BRCA (orange), COAD (yellow), ESCA (light green), GBM (green), KIRC (turquoise), LUAD (light blue), and PRAD (dark blue). The unique (fuchsia) and shared (purple) groups are depicted in the right hemisphere. The inner arcs represent the distribution of the DE-IGs in each cancer, while the outside arcs represent the intersection of both hemispheres on a scale of $0-100 \%$. Data was obtained from the TCGA database.

\subsubsection{GBM}

Among the specific DE-IGs found in GBM, an evident first group includes mainly cellular proliferation and cytoskeleton-related genes. An enrichment of microtubule-based movement, transport along the microtubule, cytoskeleton-dependent intracellular transport, and microtubule-based transport is identified $(\mathrm{FDR}=0.0132)$. Moreover, the second group of specific deregulated genes is related to tumor suppression, a negative regulatory mechanism of cell growth control, which usually inhibits tumor development. We identified GBM specific deregulated genes with crucial involvement in the $p 53$ pathway, such as KLLN, INSM2 and SFN.

\subsubsection{KIRC}

The cancer-specific deregulated IGs in KIRC include the tissue-specific KAAG1 gene (kidney-associated antigen 1) along with transcription factors POU5, TAL, and $b H L H$. Moreover, transmembrane proteins such as the protocadherin beta 10 (PCDH10), related to the Wnt 
signaling, were also upregulated. $P C D H 10$ has been classified as a tumor suppressor in multiple cancers. It induces cell cycle retardation and increases apoptosis by regulating the $p 53 / p 21 / R b$ axis and $B c l-2$ expression [17].

\subsubsection{PRAD}

IGs specifically downregulated in PRAD include RAB6D (member RAS oncogene) and MARS2 (methionyl-TRNA Synthetase 2) related to amino acid metabolism, while PBOV1, a 135 amino acid protein with a transmembrane domain was highly upregulated $(\log F C=3.1699)$. This gene is mapped to 6q23-q24, a region associated with loss of androgen dependence in prostate tumors [18]. Other upregulated IGs include transcription factors such as FOXB2 (Forkhead Box B2), and $A T O H 1$ a $b H L H$, which is associated with goblet cell carcinoid and Merkel cell carcinoma diseases (Verhaegen et al 2017). Moreover, FRAT2, an essential positive regulator of the Wnt signaling and $L E N G 9$, a member of the conserved cluster of receptors of the leukocyte-receptor complex (LRC) were also upregulated [19].

\subsubsection{BRCA}

Few cancer-specific differentially expressed IGs that code for TFs were identified for BRCA, while specific gene expression is more abundant for transmembrane proteins in this cancer, such is the case of a group of glycoproteins and transmembrane receptors involved in signal transduction. For instance, upregulation of PIGM (phosphatidylinositol glycan anchor biosynthesis class M), GP5 (glycoprotein V platelet,) CALML5 (calmodulin-like 5), coiled-coil domain-containing proteins $C C D C 96$, and $C C D C 87$, are identified specifically for this cancer.

\subsubsection{LUAD}

Regarding the specific differential expression found for lung tumors, the following TFs are significantly upregulated: $T A F 1 L$, a transcription factor that is related to chronic inflammatory diseases by regulating apoptotic pathways including regulation of TP53 activity, and FOXE1, a forkhead TF previously related to thyroid cancer [20]. Interestingly, specific transmembrane signaling receptors are upregulated. $M A P 10$ is a microtubule-associated protein and THBD thrombomodulin endothelial-specific type I membrane receptor that binds thrombin. Among $T H B D$ related pathways are collagen formation and formation of fibrin clot, as well as calcium ion binding and cell surface interactions at the vascular wall.

\subsubsection{BLCA}

In BLCA, only ZXDB (Zinc Finger X-Linked Duplicated B) TF is identified as specifically deregulated. Transcripts for signal transduction proteins are specifically upregulated for OR52E8 (Signaling by GPCR), LRRC10B (Leucine-Rich Repeat Containing 10B), and MAS1L, a proto-oncogene which is a peptide ligand-binding receptor linked to sensor neurons for pain stimuli detection. 


\subsubsection{ESCA \& COAD}

No specific TFs are specifically differentially expressed for colon and esophageal cancer. Gastrointestinal adenocarcinomas of the tubular gastrointestinal tract, including esophagus, stomach, colon, and rectum, share a spectrum of genomic features, including TF-guided genetic regulation [21]. In keeping with this, we found that transmembrane proteins related to GPCR signaling pathways are specifically undergoing differential expression for both cancers; for instance, in colon, GPR25 (G protein-coupled receptor 25), CCDC85B (coiled-coil domain containing 85B), CCDC184 (coiled-coil domain containing 184), SPRR1A (small proline-rich protein 1A), PROB1 (proline-rich essential protein 1). Meanwhile, for esophagus tumors, OR51B4 related to signaling by GPCRs, and $L D H A L 6 B$ involved in glucose metabolism and respiratory electron transport were identified.

Remarkably, when comparing IG's deregulation across the eight cancer types, we identified that ESCA and LUAD shared $93 \%$ of the DE-IGs, while BRCA, BLCA, COAD, PRAD, and KIRC shared more than $86 \%$ at least with another cancer. Finally, and presumably given its "multiforme" nature, GBM is the cancer type identified with a significant but lesser percentage of shared DE-IGs $(\sim 79 \%)$. Notwithstanding, our results show that GBM represents the tumor where the IGs have more differential expression, specificity, and concerted functional roles. This could be explained due to their gene expression tissue-specific relevance in the brain.

\subsection{Proteins encoded by cancer-specific deregulated IGs interact with distinct groups of proteins in PPI networks}

Gene expression is a phenomenon where coupled biochemical interactions take place to transcribe mRNA for protein production. There is a high regulation in the balance of this event. Hence differences in protein composition, production, or abundance are a consequence of disruptions in cell phenotypes. The differential expression pattern of the mRNA is a key in determining a cell state at the molecular and physiological level [22]. It has been shown that genes involved in "similar diseases" depict shared protein-protein interactions (PPI), and higher expression profiling similarity [23].

To determine if the DE-IGs play a crucial role in inhibiting or exacerbating biological reactions at a physical level, we obtained the Protein-Protein Interactions (PPIs) with highest confidence where DE-IGs for each cancer are involved. Our results show that a considerable proportion of proteins encoded by DE-IGs in each cancer interacts with specific groups of proteins, due to the low percentage of DE-IGs that are shared between two or more cancers (Supplementary Figure 5). In this work, we will consider that an interaction is shared between cancers, if a DE-IG in one cancer is also deregulated in another cancer, and the interaction of the IG-encoded protein with another protein is reported by String, otherwise, we classify that interaction as cancer-specific. 
For instance, $100 \%$ of the interactions found in LUAD are cancer-specific, followed by the high specificity of interactions found in ESCA (99.3865\%) with only a unique shared PPI of centromere complex with BLCA (HIST1H2BJ, CENPA). GBM has a similar pattern with $95.8333 \%$ of unique interactions and only 19 shared ones. In BLCA, $59.16 \%$ of the analyzed PPI are specific to this cancer type. PRAD is a condition that possesses $53.1339 \%$ cancer-specific interactions; and COAD has $42.1455 \%$. For BRCA, $30.62 \%$ of its interactions are only found in this cancer, the lowest fraction of unique interactions. KIRC has the second-lowest with only $31.55 \%$ of unique interactions.

If two cancers share PPIs related to the cancer-specific DE-IGs, there could be an underlying affected process characteristic for such diseases. To determine those specific processes, protein complexes involved in shared interactions were examined, finding that most of the identified proteins belong to families of core histones. Overall, the analyzed PPIs indicate a very distinct pattern of interactomics for each cancer. The tumors that share the greatest proportion of PPIs are breast and colon, having 145 common interactions representing $44.61 \%$ of the total interactions identified for such diseases. The second higher similarity found is for prostate and kidney tumors, sharing 227 interactions $(28.64 \%$ of all). The rest of the tumors share at most $21.32 \%$ interactions (Supplementary Figure 5, Supplementary Table 4).

These cancer-specific interactions were analyzed deeper to delve into their functional role in each cancer (Figure 3). As it could be expected due to their high specificity at the PPI level, this network approach shows specific clusters well defined for glioblastoma (Fig 3b) and lung (Fig 3d). In less-defined clusters, esophageal and bladder cancer can be observed in panels e) and f). In contrast, in the case of prostate, bladder, and kidney tumors, the interactions are linked by common DE-IGs in a cluster. On the other hand, we identified that the communities in the network are defined primarily by the type of proteins. Functional enrichment shows specific biological processes intrinsic to the physical interactions implied for the genes in each cancer (Supplementary Figure 6). For instance, proteins involved in BLCA-specific PPIs are conducting mainly chromatin organization and DNA repair reactions, while in colon tumors, proteins play a concerted role in the regulation of transcriptional processes. In keeping with this, in esophageal tissue, the interactors are involved in the regulation of different classes of non-coding RNAs. Meanwhile, in glioblastoma and lung tumors, proteins are specifically interacting for splicing activity, and transcription initiation processes, respectively. Kidney and prostate tumors show specific protein-protein interactions for DNA-replication and protein-protein complex formation, and DNA organization and packaging. In contrast, proteins with specific interactions in breast tumors, have an important activity linked to cellular growth and apoptotic processes.

Intrinsic of the PPI networks is the topological information capable of characterizing cancer proteins [24]. Surprisingly, our comparison of IGs with their physical interactors at a protein-protein level shows a tendency to lower betweenness centrality, lower degree, and a few interactions with other IGs. Therefore, even though IGs are not hubs in the network of interactions where they participate, changes in their regulatory role can cause a cascade of disruptions in interactions that might lead to malignancy. 


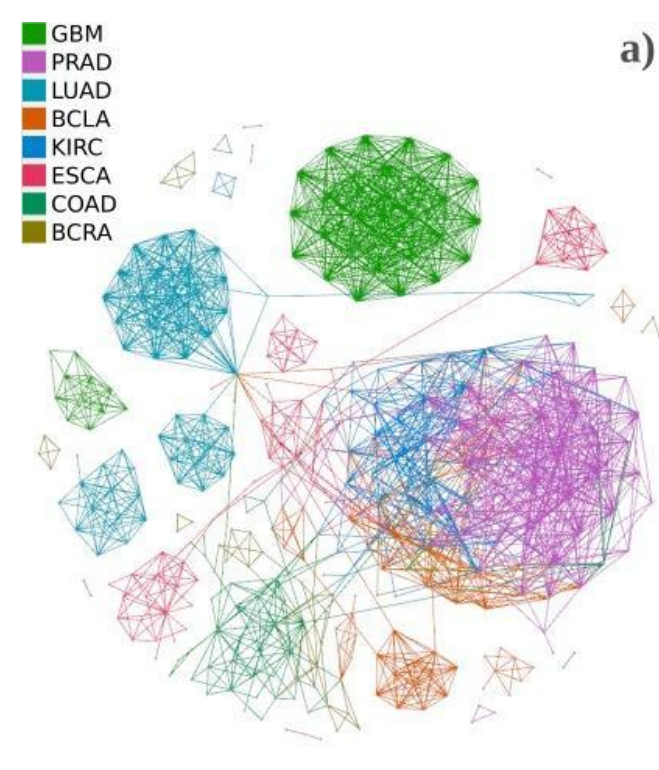

a)

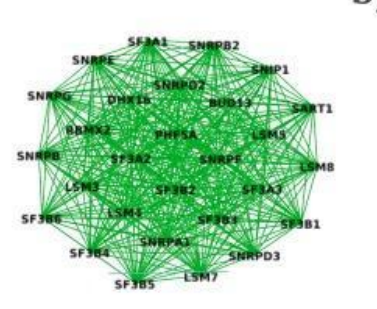

b)

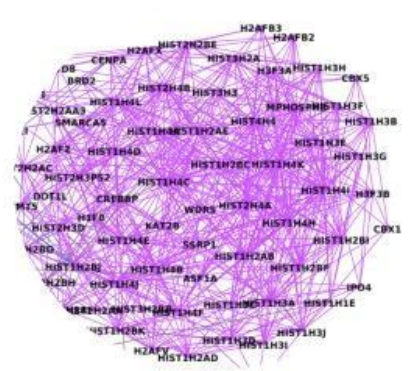

e)

c)

d)

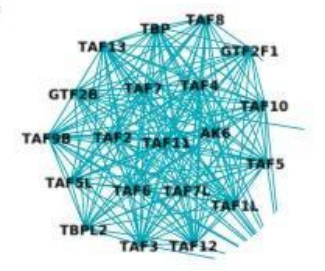

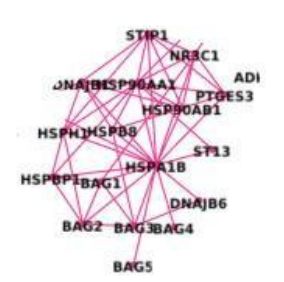

Figure 3. Protein-protein interaction network of DE-IGs and their 50 closest interactors across cancers. a) Unique and shared PPI across cancer are shown, each cancer has a specific color for its interactions. Close-ups of the biggest and more isolated clusters in the network are presented: b) GBM, c) PRAD, d) LUAD, e) ESCA, and f) BLCA. Data was obtained from the String database.

Altogether, these results are the opposite of the patterns reported for cancer genes, which suggests that the behavior tendency of IG proteins is to interact with oncogenic genes [24].

For a closer approach, the nearest fifty interactors of the DE-IG encoded proteins in each cancer were analyzed. In Figure 4 we can observe that cancer-specific DE-IG encoded proteins interact with specific groups of proteins in distinct cancers, suggesting that DE-IGs affect particular post-transcriptional processes.

The most important protein complexes for each type of cancer were determined for cancer-specific deregulated IGs and their interactors (Supplementary Table 5). In total, we detected 27 protein complexes where cancer-specific DE-IGs and their interactors are found. Colon cancer is the disease containing more DE IGs in the same complex: 9 out of 13 corresponding to the disassembly of the beta-catenin Wnt signaling pathway. Bladder DE IGs are interacting mainly with the proteins of the CD40 signaling pathway, and are involved in multi-multicellular organism processes. Likewise colon, breast cancer-specific IGs are related to wnt signaling pathway, but also to follicle-stimulating hormone signaling. Glioblastoma-specific proteins have a major participation in splicing, and vesicle transport. Kidney's are more related to regulation of feeding behavior. Esophageal's IGs are involved in protein folding, and tRNA and ncRNA processing. Lung cancer proteins participate in transcription preinitiation complex assembly, as well as in the BMP signaling pathway. Prostate cancer DE-IGs are found mainly related to chromatid sister cohesion and translation and postranslation processes . The protein complex related to Wnt signaling pathway is associated with a large group of deregulated genes among Bladder, Breast, Colon, Kidney and Glioblastoma, being a total of 17 IGs with disrupted expression. 

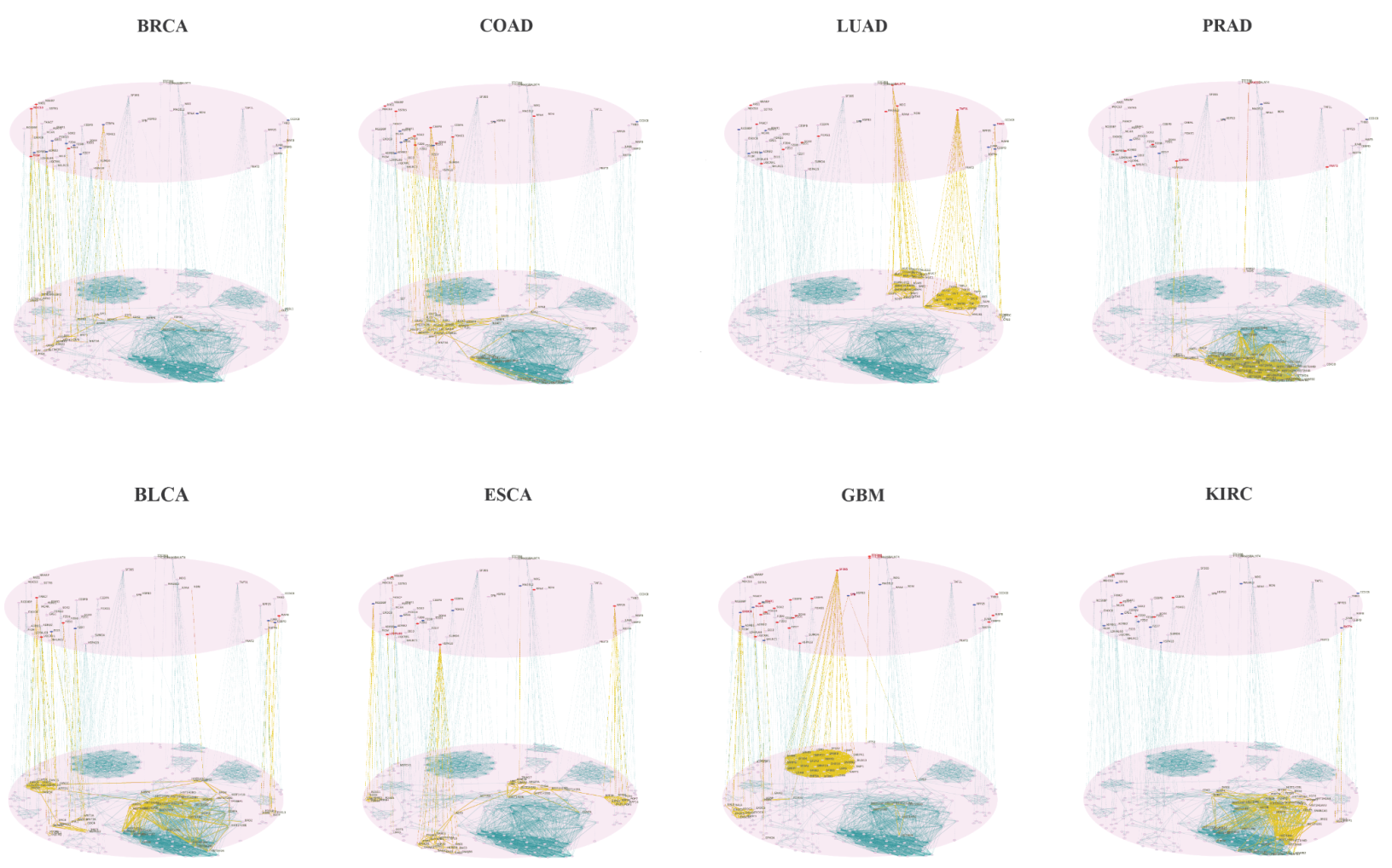

Figure 4. Multilayer networks of IG-encoded proteins and their cancer-specific interactors. Protein-protein interactions were found for deregulated IGs in each cancer. The cancer-specific deregulated IGs that encode for proteins interact with exclusive groups of proteins in each cancer. Upper layer shows deregulated IGs across cancer, highlighting the cancer-specific upregulated IGs in red,) and downregulated in blue. Links to the bottom layer connect IG-encoded proteins to proteins found in PPIs by String. Yellow links highlight interactions that can be affected by deregulation of IGs in a specific type of cancer.

\subsection{DE-IGs participate in the genetic "rewiring" of cancer cells}

Cancer cells undergo significant genetic "rewiring" as they acquire metastatic traits and adapt to survive in multiple environments with varying nutrient availability, oxygen concentrations, and extracellular signals. Therefore, to effectively treat metastatic cancer, it is important to understand the strategies that cancer cells adopted during the metastatic process. Finally, we focus on studying the "rewiring" between healthy and tumor samples using BRCA as a model (Figure 5). We used breast cancer since it is the only dataset that fits the criteria for a mutual information analysis (at least 100 samples for each condition are required). A network of DE-IGs and their co-expressed genes was built and analyzed for each condition. Our results show that the tumor co-expression network is composed of a total of 62,462 interactions among 15,347 genes, while the healthy network has 13,037 genes with 45,941 interactions. There are only 9,615 interactions shared between the two network topologies. All the differences among these two networks are potential rewiring-caused co-expression interactions that may be part of the mechanism that BRCA cells follow to achieve the characteristics of their phenotype. For instance, as seen in Figure 5, IGs like RRS1, FNDC10, EPOP and $N N D$ are highly affected by this "rewiring" behavior in cancer. According to differential expression and network analysis, 
we observe that histones play a key role in rewiring the co-expression mechanism from healthy to cancer tissue. Examples of this are $\mathrm{H} 2 \mathrm{AW}$ and $\mathrm{H} 2 \mathrm{BC} 8$, which are core histones that have only few healthy tissue-specific interactions that are almost lost during cancer development, which creates many new interactions with other genes.

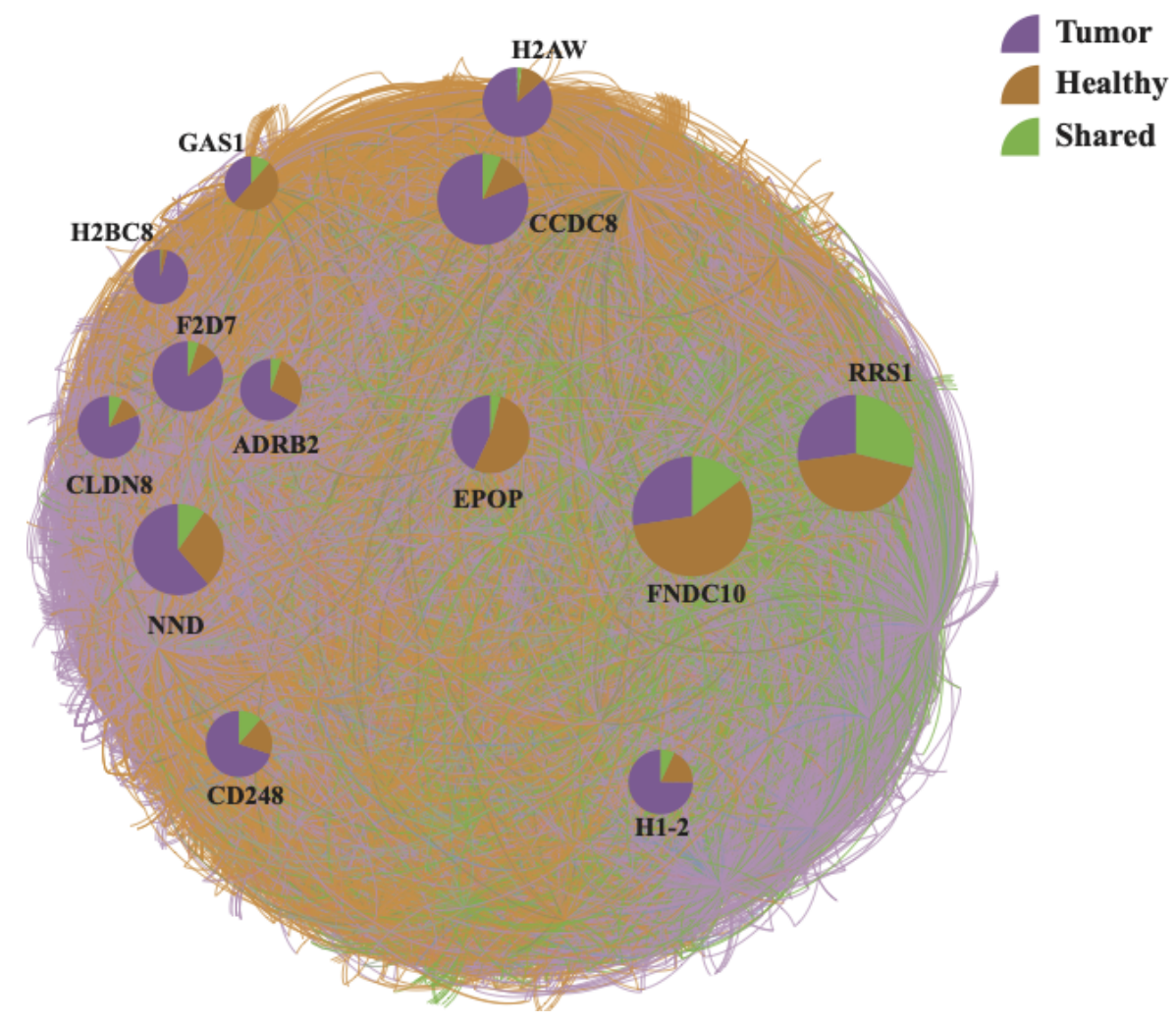

Figure 5. Co-expression network of healthy and tumoral breast tissue. In this network, the co-expression of DE-IGs found in BRCA in healthy tissue (depicted by brown links) and breast tumor tissue (purple links) is shown. Shared co-expression relations are depicted with green links. Pie charts for IG nodes of a higher degree are shown. Each of them indicates the percentage of each type of interaction: in purple tumor-specific interaction, in brown healthy-specific interaction, in green conserved interaction. We can see the effect of rewiring, by observing that a high percentage of interactions that are present in healthy tissue is not observed in tumor tissue. Moreover, many more new interactions emerge in the tumor tissue.

\section{Discussion}

Here, we present a comprehensive analysis of differentially expressed Intronless genes (IGs) across eight cancers using a standardized bioinformatics pipeline. We uncover signature changes in gene expression patterns of IGs such as enhanced expression changes when compared to multi-exon genes, unique and shared transcriptional signatures between different cancer types, and association with specific protein-protein interactions that might have potential effects in post-transcriptional processes. 
Strikingly, our results show that the upregulated IGs across the eight different cancer types were related to negative regulation of gene expression (gene silencing) and negative regulation of cell differentiation. Further, we found a core set of chromatin-modifying genes such as HASPIN and Histone deacetylases (HDACs), located on chromosome 6, that were upregulated across all the analyzed cancers. Although the pattern of differentially expressed (DE) IGs was found to be not specifically shared across the different tumor types, highlighting their specificity modulating cancer signaling and proliferative pathways. For example beta-catenin Wnt signaling was regulated in kidney and prostate tumors while GPCR signaling critical to inflammation [25-27] was regulated in gastrointestinal esophageal and colon adenocarcinomas, and p53 pathways.

This biological behavior could be due to tumor's cellular origin and heterogeneity [28], and tissue-specific expression $[2,8]$. Due to their remarkable heterogeneity, glioblastoma, prostate, and kidney tumors were found with a less representative shared behavior in the upregulation of IGs. Notably, glioma tumors are the malignant microenvironment where IGs showed the most characteristic gene expression regulation profile with 35 unique DE-IGs, more than 50\% greater than other cancers. Notwithstanding, our results show that GBM represents the tumor where the IGs have more differential expression, specificity, and concerted functional roles. This could be explained due to the "multiforme" nature of GBM that is driven by brain-specific gene expression as is further evidenced by least shared DE-IGs $(\sim 79 \%)$ with other cancers.

In conclusion, IGs drive massive transcriptional rewiring, as observed in co-expression analysis, that may drive tumorigenesis and may act as novel therapeutic targets for gene therapy and in-silico drug design such as popular GPCRs or emerging HDAC inhibitors [29].

\section{Materials and Methods}

\subsection{Data extraction and curation for IG, and MEG datasets}

Data was extracted using Python scripts (https://github.com/GEmilioHO/intronless genes), and the Application Programming Interface (API). Homo sapiens genome was assembled at a chromosome level and was accessed at the Ensembl REST API platform (http://rest.ensembl.org/ accessed using Python with the ensembl_rest package). The pipeline process was as follows: protein-coding genes with CDS identifiers for transcripts for all chromosomes were retrieved and classified into two datasets named "single-exon genes" (SEGs), and "multiple exon genes" (MEGs) depending on exon and transcript count. The SEG dataset was submitted to the Intron DB comparison (http://www.nextgenbioinformatics.org/IntronDB) to separate those with UTR introns and referred to as uiSEGs. The output of the pipeline was a third dataset containing only intronless genes (IGs). After data extraction, a manual curation step in the IG and uiSEG datasets was followed to discard incomplete annotated protein sequences and mitochondrial encoded proteins. The final IG dataset contained 940 protein-coding genes with only one exon and one transcript. 


\subsection{Bipartite network \& quantification of shared and unique DE-IGs}

The bipartite network was constructed with upper nodes representing IGs and bottom nodes representing cancer types. We place links connecting each IG to the cancer types where such a gene is differentially expressed (DE). Nodes corresponding to genes in the bipartite network were sorted by degree aimed to visually identify intronless genes cancer-specific deregulated, and those whose expression is disrupted in most of the diseases. A heatmap for shared IGs is computed by identifying the number of deregulated IGs in every pair of cancers, and dividing such quantity by the total of the disrupted genes in any of the compared diseases. This metric is known as the Jaccard similarity coefficient.

\subsection{Data source and differential expression analysis across cancer}

Currently, the (The Cancer Genome Atlas) TCGA possesses data for the study of 37 different tumor types. We found the primary tumor and adjacent normal tissue transcriptomic data for 18 types. Among those, we selected 8 types for the present study. Gene expression data from patients for BRCA, BLCA, COAD, ESCA, GBM, KIRC, LUAD and PRAD cancers was downloaded from the NIH website (https://portal.gdc.cancer.gov/) using the TCGAbiolinks R package [30] with the following restriction criteria: samples types primary tumor and solid tissue normal (control); results of RNAseq experimental strategy; and workflow type HTSeq-counts format. Differential expression analysis was carried out using the TCGAutilis R package [31], indicating which of the obtained samples correspond to tumors and which to control, and establishing a filtering threshold of FDR $=0.05$ and $\log \mathrm{FC}=1$ (absolute value) to consider a gene significantly differentially expressed.

\subsection{Functional Enrichment Analysis of Differentially Expressed IGs}

The functional enrichment was conducted using the over-representation analysis of the functional assignment (ORA). Genes with differential expression up to one log2-fold change values were considered as up-regulated with a $p$ - and $q$-value set at 0.05 and 0.10 , respectively. First, the functional enrichment of the 338 differentially expressed human IG proteins (up-regulated and downregulated separately) was performed using all human IGs proteins as a background "universe" (selecting input as species: Homo sapiens, universe 940 human IGs). The comparative functional enrichment analyses were performed using Metascape (https://metascape.org/) for the biological process category, including KEGG and Reactome pathways. To delve insight into the role of specific IGs in the affected biological processes ORA was assessed to determine category barplots. The Circos software [32] was employed for data analysis and visualization.

\subsection{DE-IGs PPI network construction \& protein complex identification}

Network analysis and visualization were performed using python scripts (See repository) and the Gephi software. StringDB platform [33] was used to download physical and functional interactions data. The highest confidence scores (0.9) were filtered for this study, keeping the most probable interactions. Then, interactions were requested for the set of unique DE-IG of each cancer type, downloading relations between those genes, and also their interactors in the 
first shell (up to 50 interactors). All this data is assembled into a single network. Network metrics such as degree distribution, closeness, and betweenness centralities were computed using Python networkx library [34]. For protein complex detection, we assumed that highly clustered nodes in the PPI network correspond to protein complexes. Therefore, 27 protein complexes were predicted using the Louvain method to find communities implemented in Gephi.

\subsection{BRCA network deconvolution}

Co-expression networks were inferred using ARACNe-AP [45], a mutual information-based tool for gene regulatory network deconvolution. In this analysis, we used separated submatrices for healthy and tumor tissue and the list of every DE-IG in the BRCA dataset. For this study, a $p$-value of $1 \times 10^{-8}$ was set up and 100 bootstraps were carried out. Then, they were consolidated into a single network, getting an inferred co-expression network for each condition.

Acknowledgments: We would like to thank Roddy Jorquera and Carolina González for fruitful discussions. Special thanks to Fernando Flores for helping with designing and constructing the co-expression network. We are also thankful to Carlos González for visualization and technical support.

This research was funded by Conacyt Ciencia Basica Project 254206. K.A.P (CVU:227919), J.A.R.R (CVU: 1147711), and O.Z.M (CVU: 1147042) received financial support from CONACyT. K.A.P is a current holder of a fellowship from the Fulbright Comexus García-Robles foundation. 


\section{Appendix}
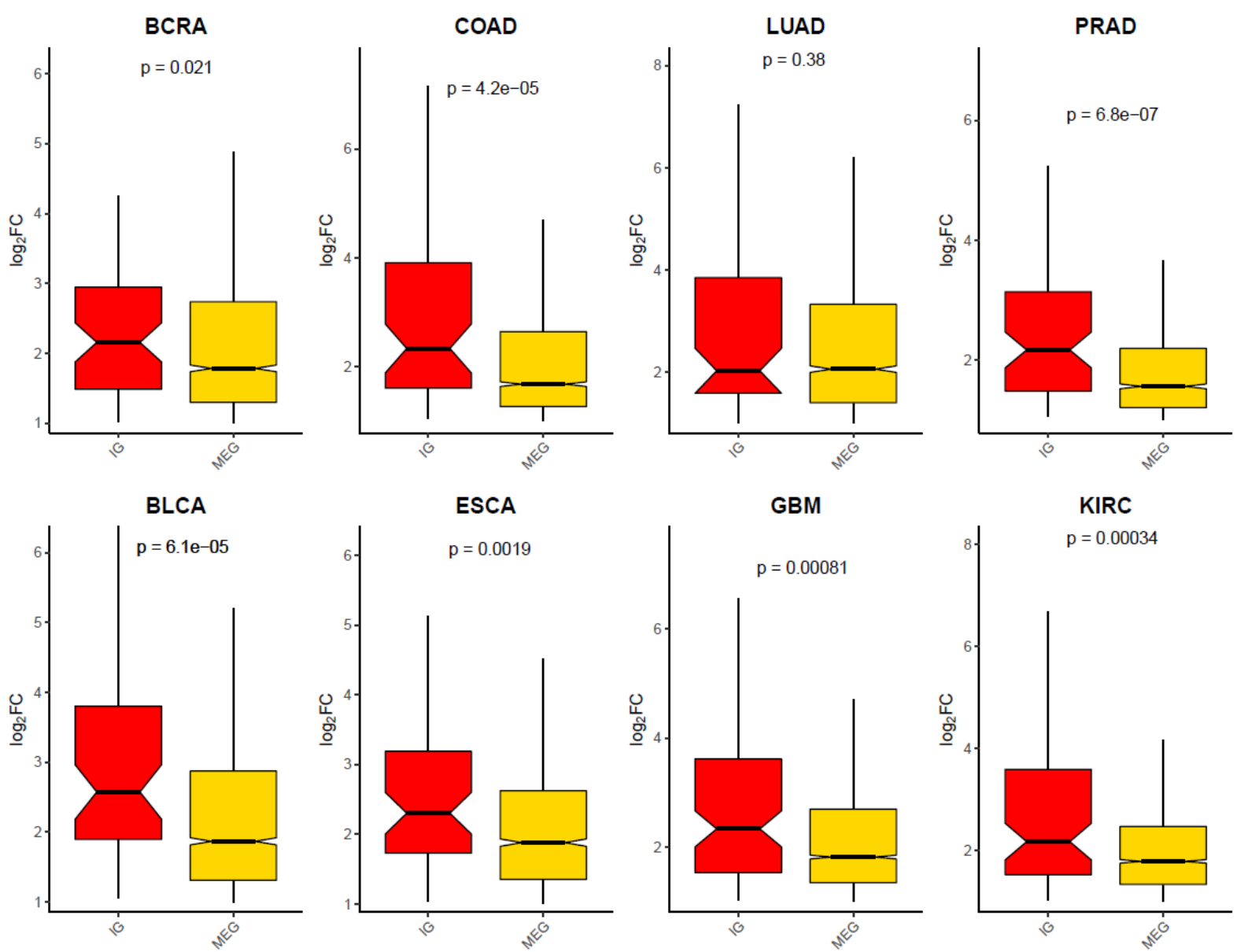

Supplementary Figure 1. Upregulated gene expression ranges among cancers. A boxplot is shown for each cancer type, separated by gene type (IGs or MEGs); expression ranges are shown on the $\mathrm{y}$-axis using $\log 2$ fold change values. The boxes present a notch corresponding to the confidence interval for each population. In the upper part of the graph, the $p$-value for the Wilcoxon test is shown in order to indicate the statistical difference between both populations. 


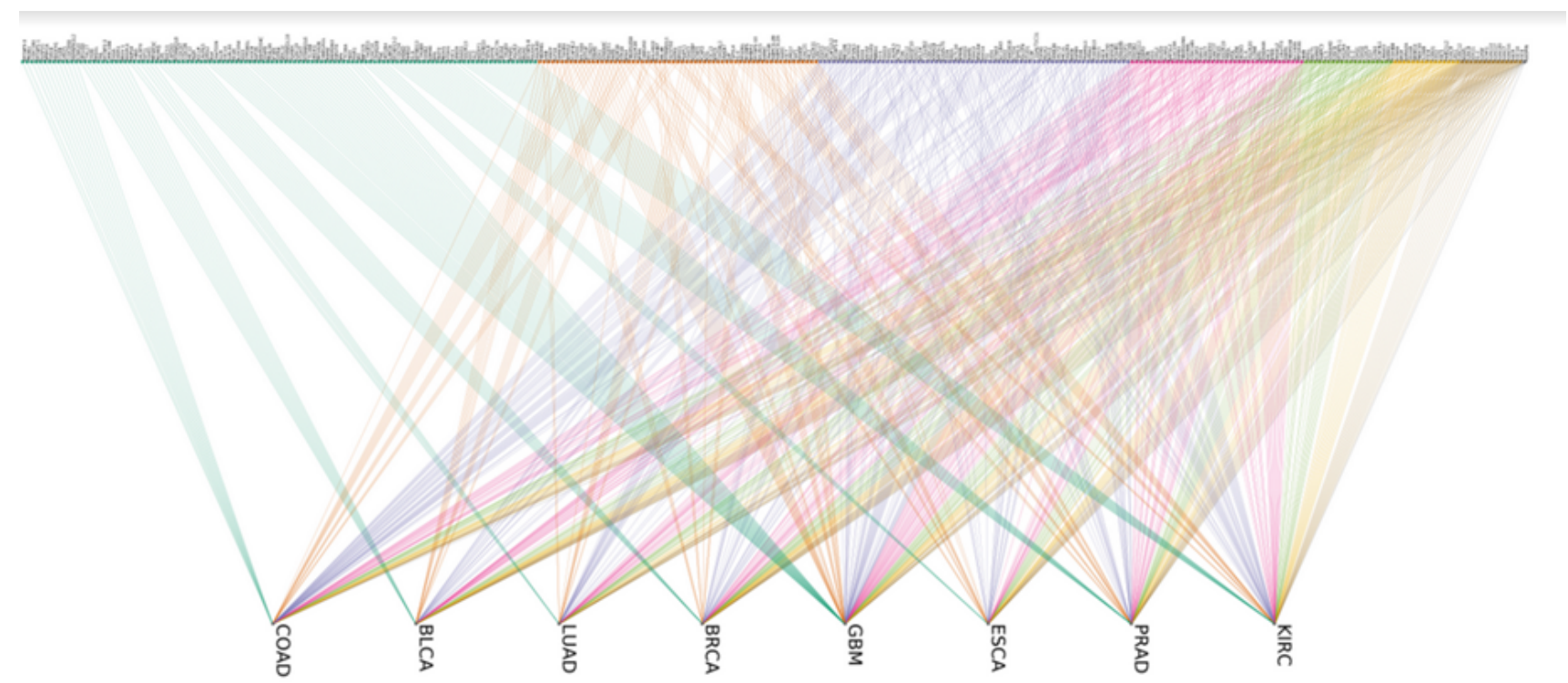

Supplementary Figure 2. IG-cancer type bipartite network. Intronless genes are shown in the upper group of nodes, while cancers are located in the bottom group. A link joining an IG and a cancer type indicates that the expression level of the IG is found disrupted in the linked cancer type. Upper nodes are color-sorted by their cancer specificity: genes deregulated in a single cancer are shown at the left in blue, followed by genes deregulated in two cancers, tree cancers, and so on. At the right of the upper nodes, there is a single gene (HASPIN) whose expression is disrupted in all tissues. 


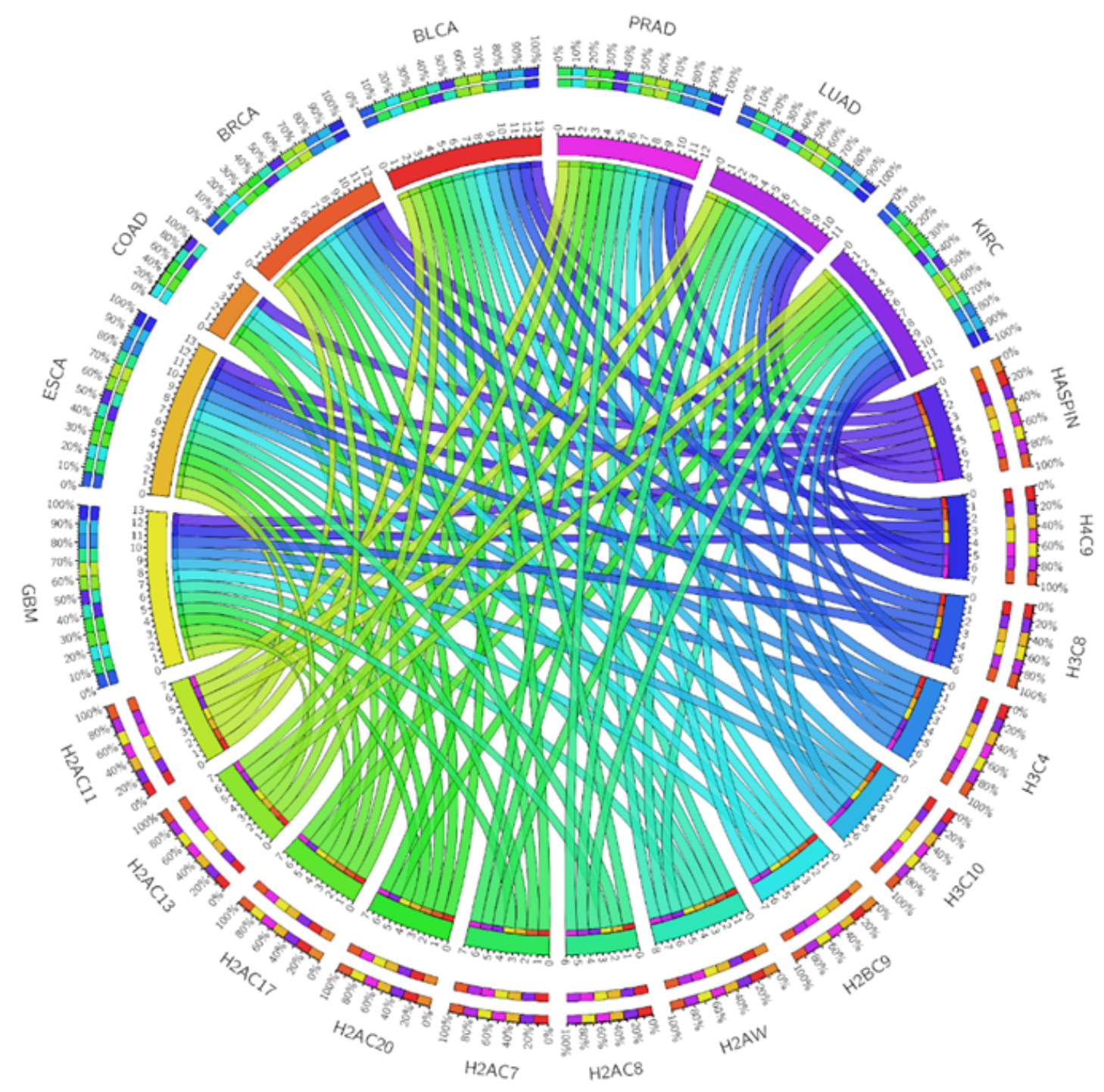

Supplementary Figure 3. A high conserved group of up-regulated histones across tumors.

Circa plot depicts the conservation of the deregulation of a group of histones across cancers. In the upper hemisphere, the total number of histone-related DE-IGs in each cancer is represented by an arc: GBM (yellow), ESCA (mustard), COAD (orange), BRCA (dark orange), BLCA (red), PRAD (pink), LUAD (violet), and KIRC (purple). Histone encoded IGs are depicted in the lower hemisphere. The inner arcs represent the distribution of the histones in each cancer (0-13 genes), while the outside arcs represent the tumors that have that particular gene upregulated (on a scale of $0-100 \%$ ). Data was obtained from the TCGA database 
a)

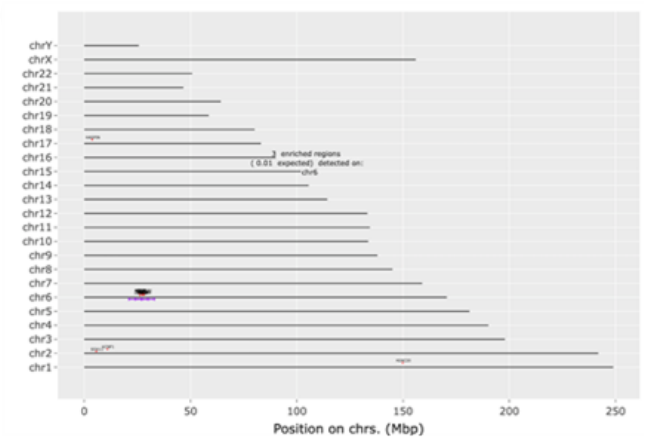

b)

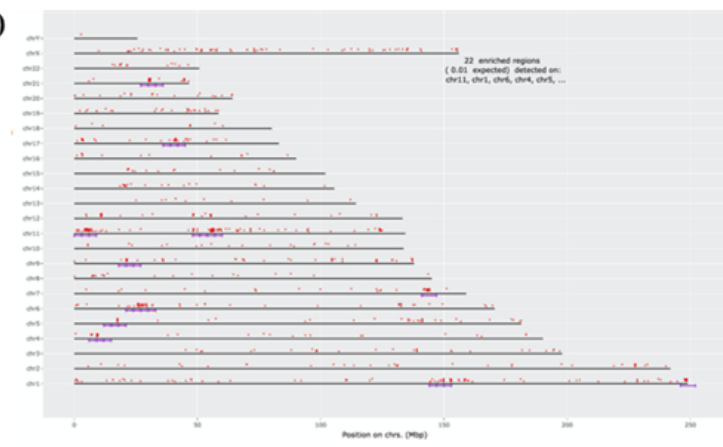

Supplementary Figure 4. Comparison of genome location of highly conserved DE-IGs a) Genome location of IGs in the human genome. b) Genome location of highly conserved DE-IGs IGs encoded proteins are represented by red dots. The purple lines indicate regions where these genes are statistically enriched, compared to the density of genes in the background. The hypergeometric test is used to determine if the presence of the genes is significant. Essentially, the genes in each region define a gene set/pathway. The chromosomes may be only partly shown as the last gene's location to draw the line is used. Data was obtained from the ENSEMBL database.

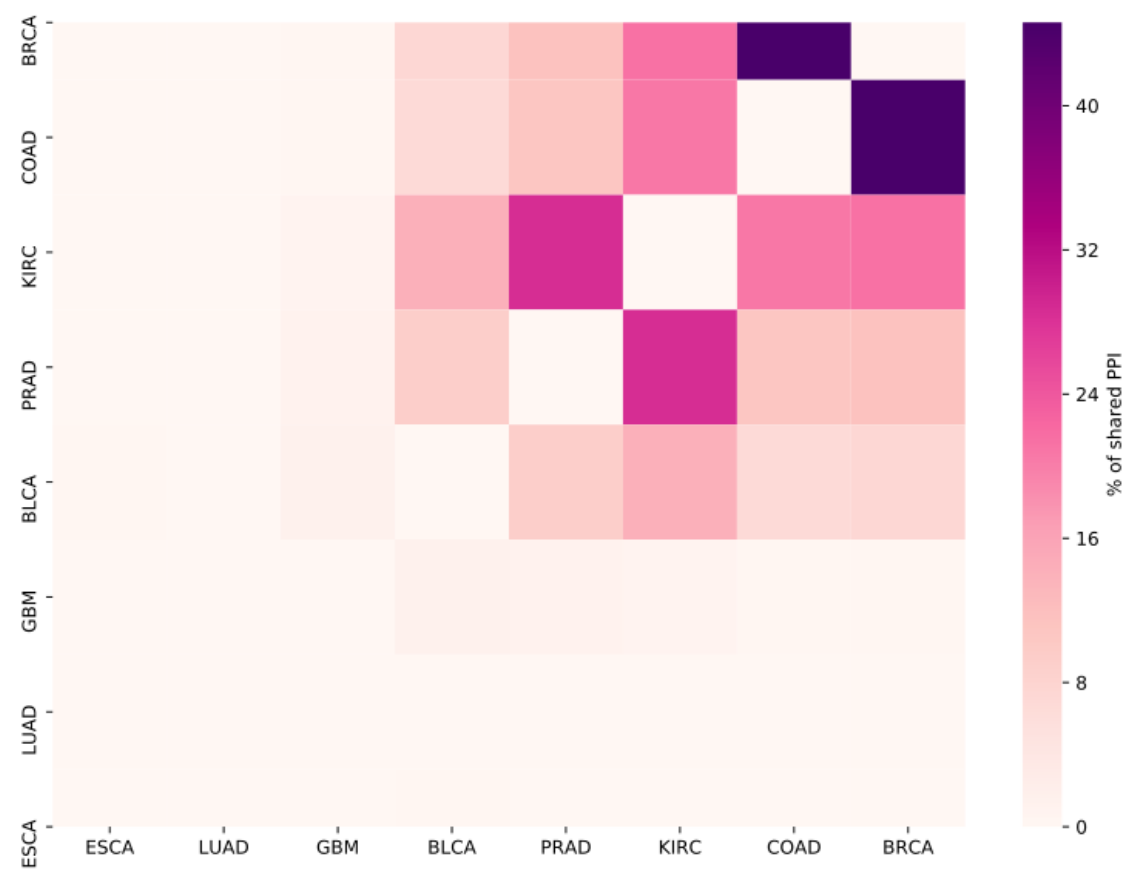

Supplementary Figure 5. Shared PPI heatmap. Color intensity indicates a higher percentage of shared PPI related to DE IGs of the compared diseases. LUAD does not share any DE IG interaction with other cancer types, and ESCA only one with BCLA. GMB shares almost no interaction with any other cancer (a maximum of $1.64 \%$ with BLCA). COAD and BRCA are the two cancers where more DE IGs are shared, therefore having more interactions in common. 


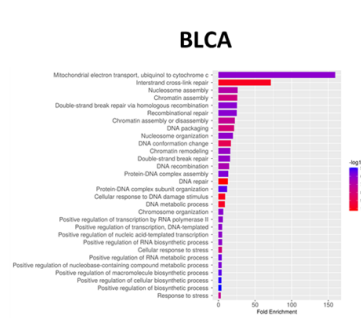

GBM
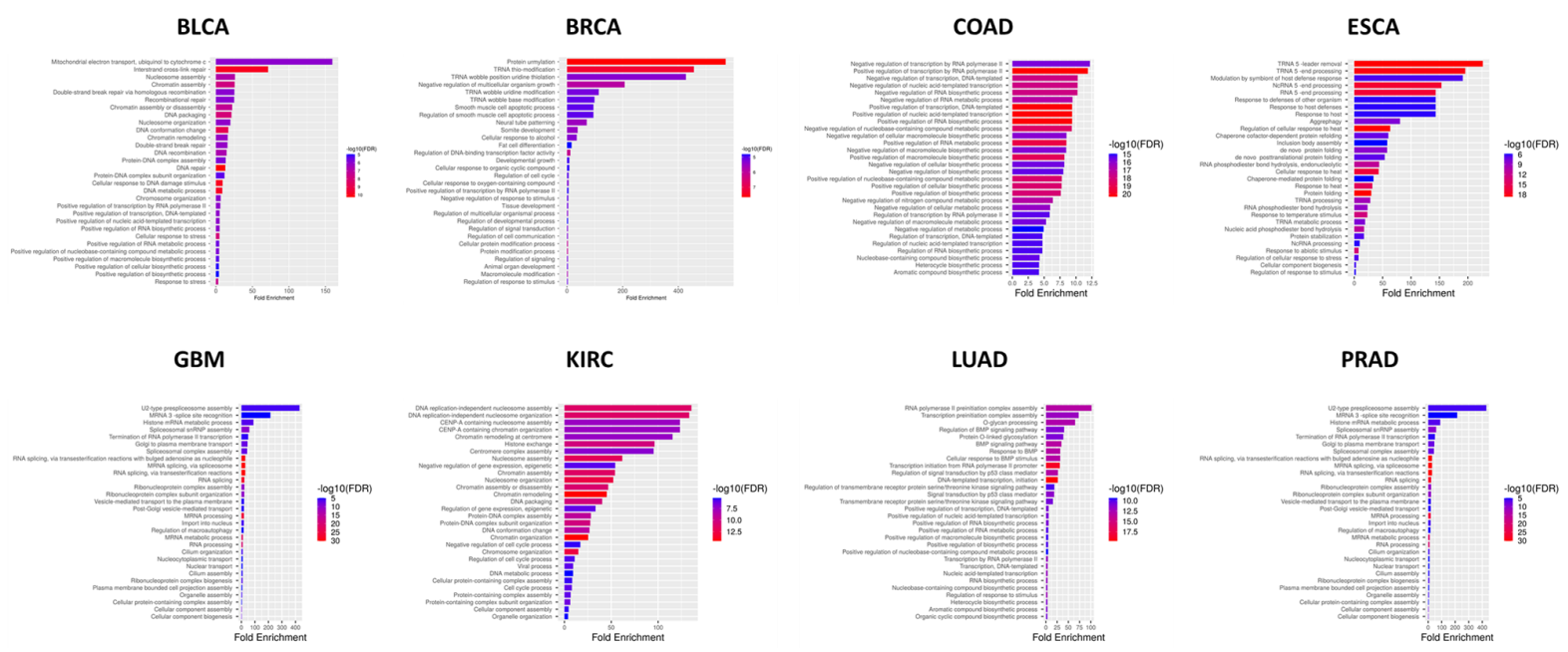

Supplementary figure 6. PPI networks ontologies. FDR is calculated based on nominal P-value from the hypergeometric test. Fold Enrichment is defined as the percentage of genes in each cancer belonging to a pathway, divided by the corresponding percentage in the background. FDR indicats how likely the enrichment is by chance. In x-axis the Fold Enrichment indicates how drastically genes of a certain pathway are overrepresented. FDR values are depicted red (high) to blue (low).

\section{Supplementary Table 1. Available expression data for each type of cancer}

\begin{tabular}{|c|c|c|}
\hline Cancer Type & $\begin{array}{c}\text { Primary Solid Tumor } \\
\text { Samples }\end{array}$ & $\begin{array}{c}\text { Solid Tissue Normal } \\
\text { Samples }\end{array}$ \\
\hline BLCA & 414 & 19 \\
\hline BRCA & 1102 & 113 \\
\hline COAD & 478 & 41 \\
\hline ESCA & 161 & 11 \\
\hline GBM & 156 & 5 \\
\hline KIRC & 538 & 72 \\
\hline LUAD & 533 & 59 \\
\hline PRAD & 498 & 52 \\
\hline
\end{tabular}


bioRxiv preprint doi: https://doi.org/10.1101/2022.02.21.481319; this version posted February 22, 2022. The copyright holder for this preprint (which was not certified by peer review) is the author/funder, who has granted bioRxiv a license to display the preprint in perpetuity. It is made available under aCC-BY-NC-ND 4.0 International license.

\section{Supplementary table 2. GO enrichment of deregulated IGs}

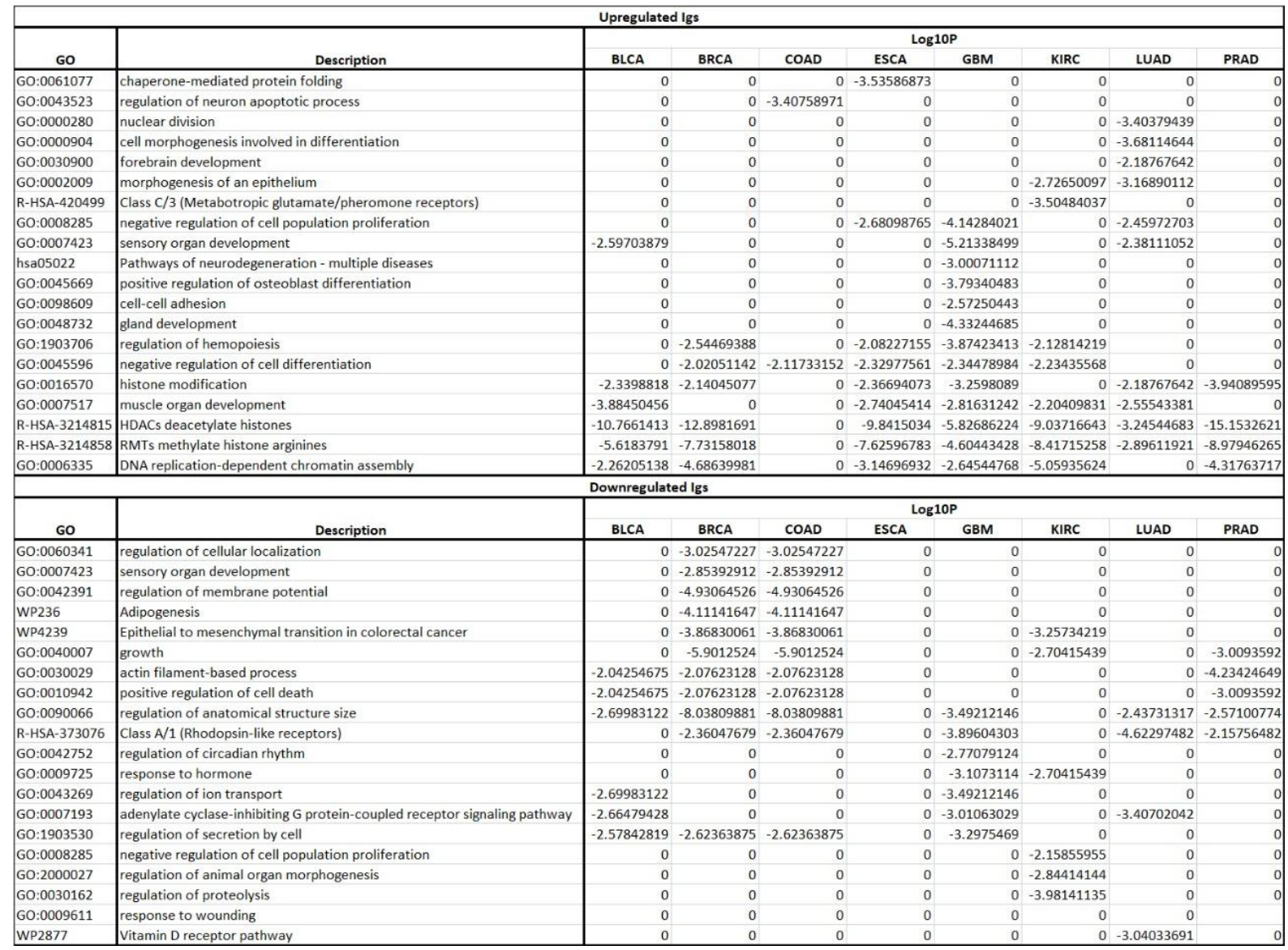


bioRxiv preprint doi: https://doi.org/10.1101/2022.02.21.481319; this version posted February 22, 2022. The copyright holder for this preprint (which was not certified by peer review) is the author/funder, who has granted bioRxiv a license to display the preprint in perpetuity. It is made available under aCC-BY-NC-ND 4.0 International license.

\section{Supplementary Table 3. Cancer-specific deregulated genes}

\section{Each column contains the list of cancer-specific DE intronless genes.}

\begin{tabular}{|c|c|}
\hline Protein complex & Coding genes \\
\hline CD40 signaling pathway & FANCL, FANCG, FANCF, FANCE, FANCC, APITD1, C17orf70, FANCM, FANCA, STRA13, FANCB, C1orf86 \\
\hline Multi-multicellular organism process & JUNB, FOSL1, FOSL2, BATF, MAFB, CEBPD, FOS, FOSB \\
\hline Transcription preinitiation complex assembly & $\begin{array}{l}\text { GTF2B, TAF13, HIST1H1C, NDN, TAF1L, TAF11, TAF6, TAF7, TAF9B, TAF12, AK6, TP53, TAF5L, TAF3, TAF7L, TAF8, } \\
\text { HIST1H1D, GTF2F1, TAF4, TAF5, TBPL2, HMGB1, TAF10, TAF2, TBP }\end{array}$ \\
\hline Electron transport chain & UQCRB, CYC1, UQCRQ, UQCRHL \\
\hline Wnt signaling pathways & $\begin{array}{l}\text { WNT5A, SRF, FZD8, KLF5, AGRP, FOXD3, CEBPB, DDIT3, RPA1, FOXG1, RPA4, SPI1, RBBP4, PAX6, NANOG, SFN, MYB, FZD7, } \\
\text { CEBPA, SMAD3, POU5F1, LRP6, ESR1, P2RY1, WNT7A, DAXX, SALL4, SOX4, DVL1, FZD2, FOXO1, FZD9, HDAC1, JUN, EP300, } \\
\text { RPA3, HSPB3, SDC4, KLF4, SMAD4, POU2F1, SOX2, MC4R, FFAR1, E2F4, TLE1, POMC, CDK2, PPARG, GNAQ, CHD7, } \\
\text { WNT3A, CTNNB1, FZD1, WNT1 }\end{array}$ \\
\hline Chromatid sister cohetion & SMC6, SMC5, UBE2I, NDNL2, EID3, SUMO4, NSMCE1, NSMCE2, PIAS4 \\
\hline Follicle-stimulating hormone signaling & GNAS, SLC9A3R1, ADRBK1, GAS1, ARRB1, SHH, ADRB2, ADRB1, PTCH1, ARRB2, CFTR \\
\hline Dendrite morphogensis & CUL7, CCDC8, OBSL1 \\
\hline tRNA modifications & CTU1, NFS1, MOCS3, CTU2, URM1 \\
\hline Protein localization to nucleolus & RPF2, EBNA1BP2, RRS1, NIFK \\
\hline GPI anchor & PIGM, PIGX, PIGV \\
\hline Hormone-mediated apoptosis & SST, SSTR5 \\
\hline Protein folding & $\begin{array}{l}\text { DNAJC2, DNAJB6, HSP90AA1, HSPB8, ST13, HIST1H2AJ, ACTB, HSPA1B, UBB, BAG5, HSPH1, PTGES3, MAGEL2, HSPBP1, } \\
\text { KDM1B, RPS27A, ACTL6A, BAG4, UBC, TRIM27, NR3C1, HSP90AB1, STIP1, DNAJB1, BAG2, BAG3, UBA52, BAG1 }\end{array}$ \\
\hline Light stimulus & GNB5, RGS11, PDE6H, PDE6C, GNAT2, RGS9, PDE6A, RGS9BP \\
\hline Vasodilatation & LDHD, LDHAL6B, GOT1 \\
\hline Heart morphogensis & NRARP, NOTCH1 \\
\hline tRNA and NcRNA processing & RPP14, RPP30, RPP21, RPP38, POP7, RPP40, RPP25, POP5, POP4, POP1 \\
\hline Splicing & $\begin{array}{l}\text { SF3B5, SNIP1, SNRPA1, SF3A1, SNRPB, SNRPE, SNRPG, SART1, SF3A3, DHX16, LSM8, SF3A2, PHF5A, SF3B6, SNRPB2, } \\
\text { RBMX2, LSM4, LSM5, SNRPF, SF3B3, SNRPD2, LSM7, SNRPD3, SF3B4, BUD13, SF3B1, LSM3, SF3B2 }\end{array}$ \\
\hline Habituation & NHLRC1, EPM2A \\
\hline Vesicle transport & RALA, EXOC6B, EXOC4, EXOC3, EXOC6, EXOC2, EXOC5, EXOC7, RALB, EXOC8, EXOC1 \\
\hline Intraciliary transport & TTC30B, IFT52, TTC30A \\
\hline Regulation of feeding behavior & RXFP1, RXFP4, INSL5, RLN3 \\
\hline BMP signaling pathway & BMP4, GATA4, SOST, BMPR2, BMP7, GDF5, BMPR1B, NKX2-5, RGMA, HFE2, BMPR1A, BMP2, BAMBI, RGMB, NOG \\
\hline Innate immune response & GALNT4, MUC15, MUC16, MUC4, MUC7, C1GALT1, MUC5AC, MUC17, MUC1, MUC13, MUC5B, MUC21 \\
\hline Blood coagulation system & PROCR, PROC, F2, CPB2, THBD \\
\hline Translation and postranslation processes & EARS2, YARS, MARS2 \\
\hline Beta-catenin destruction complex & FRAT2, GSK3B \\
\hline
\end{tabular}


Supplementary Table 4. Shared PPIs. The first column contains pairs of cancers, followed by the number of PPI related to unique DE IG of the compared diseases, finally, in the third column is shown the percentage of shared interactions. Note that two cancer may share more genes than other couples of genes but have a smaller percentage, this is due to a difference in the total number of interactions associated with the compared tumors, for example, PRAD and KIRK share 227 PPIs, representing a percentage of 28.64, while COAD and BRCA have less common interactions (145) but a bigger percentage: 44.61 .

\begin{tabular}{|l|r|r|}
\hline \multicolumn{1}{|c|}{ cancers } & Interactions shared & Percentage of total interactions in compared cancers \\
\hline COAD,BRCA & 145 & 44.6153846153846 \\
\hline KIRC,PRAD & 277 & 28.6452947259566 \\
\hline KIRC,BRCA & 132 & 21.3247172859451 \\
\hline KIRC,COAD & 138 & 20.7518796992481 \\
\hline BLCA,KIRC & 118 & 14.1656662665066 \\
\hline BRCA,PRAD & 95 & 11.6421568627451 \\
\hline COAD,PRAD & 95 & 10.9447004608295 \\
\hline BLCA,PRAD & 95 & 9.3503937007874 \\
\hline BLCA,BRCA & 43 & 7.47826086956522 \\
\hline BLCA,COAD & 43 & 6.85805422647528 \\
\hline BLCA,GBM & 14 & 1.64512338425382 \\
\hline PRAD,GBM & 15 & 1.31233595800525 \\
\hline KIRC,GBM & 9 & 0.910010111223458 \\
\hline BRCA,GBM & 2 & 0.301659125188537 \\
\hline COAD,GBM & 2 & 0.27972027972028 \\
\hline BLCA,ESCA & 1 & 0.175131348511383 \\
\hline
\end{tabular}

Supplementary Table 5. Identified PPI complexes. Protein complexes identified where cancer-specific IG-encoded proteins and their interactors play a key role.

\begin{tabular}{|c|c|c|c|}
\hline Protein complex & Enriched functions & FDR & Cancers \\
\hline CD40 signaling pathway & $\begin{array}{l}\text { Regulation of protein } \\
\text { monoubiquitination;Regulation of CD40 } \\
\text { signaling pathway }\end{array}$ & 0.018 & BLCA \\
\hline $\begin{array}{l}\text { Multi-multicellular organism } \\
\text { process }\end{array}$ & $\begin{array}{l}\text { Response to corticosterone;Multi-multicellular } \\
\text { organism process;Myeloid leukocyte } \\
\text { differentiation;Positive regulation of } \\
\text { transcription by RNA polymerase II }\end{array}$ & $\begin{array}{l}0.00001 \\
4\end{array}$ & BLCA \\
\hline Electron transport chain & $\begin{array}{l}\text { Mitochondrial electron transport, ubiquinol to } \\
\text { cytochrome c;Pons development;Pyramidal } \\
\text { neuron development }\end{array}$ & 0.0067 & BLCA \\
\hline Chromatid sister cohetion & $\begin{array}{l}\text { Positive regulation of maintenance of sister } \\
\text { chromatid cohesion;Positive regulation of } \\
\text { maintenance of mitotic sister chromatid } \\
\text { cohesion; Regulation of maintenance of sister } \\
\text { chromatid cohesion }\end{array}$ & $\begin{array}{l}0.00005 \\
1\end{array}$ & BLCA,PRAD \\
\hline
\end{tabular}




\begin{tabular}{|c|c|c|c|}
\hline Dendrite morphogensis & $\begin{array}{l}\text { Positive regulation of dendrite } \\
\text { morphogenesis;Regulation of dendrite } \\
\text { morphogenesis;Regulation of mitotic nuclear } \\
\text { division }\end{array}$ & 0.00057 & BRCA \\
\hline tRNA modifications & $\begin{array}{l}\text { Protein urmylation;TRNA } \\
\text { thio-modification;TRNA wobble position } \\
\text { uridine thiolation }\end{array}$ & $1.5 \mathrm{E}-10$ & BRCA \\
\hline Protein localization to nucleolus & $\begin{array}{l}\text { Ribosomal large subunit export from } \\
\text { nucleus;Protein localization to nucleolus }\end{array}$ & 0.0055 & BRCA \\
\hline Wnt signaling pathways & $\begin{array}{l}\text { Canonical Wnt signaling pathway; Wnt } \\
\text { signaling pathway;Cell-cell signaling by wnt }\end{array}$ & 3.9E-19 & $\begin{array}{l}\text { KIRC,COAD,BRCA,B } \\
\text { LCA,GBM }\end{array}$ \\
\hline $\begin{array}{l}\text { Follicle-stimulating hormone } \\
\text { signaling }\end{array}$ & $\begin{array}{l}\text { Desensitization of } \mathrm{G} \text { protein-coupled receptor } \\
\text { signaling pathway by } \\
\text { arrestin;Norepinephrine-epinephrine-mediated } \\
\text { vasodilation involved in regulation of systemic } \\
\text { arterial blood pressure;Follicle-stimulating } \\
\text { hormone signaling pathway;Negative } \\
\text { regulation of multicellular organism growth }\end{array}$ & $\begin{array}{l}0.00006 \\
5\end{array}$ & BRCA \\
\hline GPI anchor & $\begin{array}{l}\text { Preassembly of GPI anchor in ER } \\
\text { membrane;GPI anchor biosynthetic } \\
\text { process;GPI anchor metabolic process }\end{array}$ & $3.4 \mathrm{E}-08$ & BRCA \\
\hline Hormone-mediated apoptosis & $\begin{array}{l}\text { Hormone-mediated apoptotic signaling } \\
\text { pathway;Somatostatin receptor signaling } \\
\text { pathway;Somatostatin signaling } \\
\text { pathway;Positive regulation of T cell anergy }\end{array}$ & 0.022 & COAD \\
\hline Light stimulus & $\begin{array}{l}\text { Wnt signaling pathway, calcium modulating } \\
\text { pathway;Detection of light } \\
\text { stimulus;Phototransduction }\end{array}$ & 0.0011 & ESCA \\
\hline Vasodilatation & $\begin{array}{l}\text { Glutamate catabolic process to } \\
\text { 2-oxoglutarate;Response to transition metal } \\
\text { nanoparticle;Negative regulation of collagen } \\
\text { biosynthetic process;Regulation of angiotensin } \\
\text { levels in blood;Angiotensin maturation }\end{array}$ & 0.021 & ESCA \\
\hline Protein folding & $\begin{array}{l}\text { Regulation of cellular response to } \\
\text { heat;Chaperone cofactor-dependent protein } \\
\text { refolding;Inclusion body assembly;de novo } \\
\text { protein folding }\end{array}$ & 4.1E-08 & ESCA \\
\hline Heart morphogensis & $\begin{array}{l}\text { Coronary vein morphogenesis;Negative } \\
\text { regulation of cell proliferation involved in heart } \\
\text { valve morphogenesis;Mitral valve } \\
\text { formation;Cardiac right atrium } \\
\text { morphogenesis;Cell proliferation involved in }\end{array}$ & 0.0099 & ESCA \\
\hline
\end{tabular}




\begin{tabular}{|c|c|c|c|}
\hline & heart valve morphogenesis & & \\
\hline tRNA and NcRNA processing & $\begin{array}{l}\text { TRNA } 5 \text {-leader removal;TRNA } 5 \text {-end } \\
\text { processing;NcRNA } 5 \text {-end } \\
\text { processing;Endonucleolytic cleavage involved } \\
\text { in tRNA processing }\end{array}$ & $\mid \begin{array}{l}6.90 \mathrm{E}-0 \\
3\end{array}$ & ESCA \\
\hline Splicing & $\begin{array}{l}\text { U2-type prespliceosome assembly;MRNA } 3 \\
\text {-splice site recognition;Histone mRNA } \\
\text { metabolic process;Spliceosomal snRNP } \\
\text { assembly }\end{array}$ & $\left|\begin{array}{l}0.00000 \\
094\end{array}\right|$ & GBM \\
\hline Habituation & $\begin{array}{l}\text { Habituation;Nonassociative } \\
\text { learning;Regulation of glycogen (starch) } \\
\text { synthase activity }\end{array}$ & 0.012 & GBM \\
\hline Vesicle transport & $\begin{array}{l}\text { Golgi to plasma membrane transport;Vesicle } \\
\text { docking involved in exocytosis;Vesicle } \\
\text { docking;Exocytic process }\end{array}$ & $\left|\begin{array}{l}0.00000 \\
085\end{array}\right|$ & GBM \\
\hline Intraciliary transport & $\begin{array}{l}\text { Intraciliary anterograde transport;Negative } \\
\text { regulation of myotube } \\
\text { differentiation;Intraciliary transport }\end{array}$ & 0.019 & GBM \\
\hline Regulation of feeding behavior & $\begin{array}{l}\text { Positive regulation of feeding } \\
\text { behavior;Regulation of feeding } \\
\text { behavior;Positive regulation of } \\
\text { behavior;Negative regulation of myotube } \\
\text { differentiation }\end{array}$ & 0.0005 & KIRC \\
\hline $\begin{array}{l}\text { Transcription preinitiation } \\
\text { complex assembly }\end{array}$ & $\begin{array}{l}\text { RNA polymerase II preinitiation complex } \\
\text { assembly;Transcription preinitiation complex } \\
\text { assembly }\end{array}$ & $\begin{array}{l}2.90 \mathrm{E}-1 \\
5\end{array}$ & BLCA,LUAD,GBM \\
\hline BMP signaling pathway & $\begin{array}{l}\text { Pharyngeal system development;Endocardial } \\
\text { cushion development;Positive regulation of } \\
\text { bone mineralization;Negative regulation of } \\
\text { BMP signaling pathway }\end{array}$ & $8.8 \mathrm{E}-12$ & LUAD \\
\hline Innate immune response & $\begin{array}{l}\text { Innate immune response activating cell surface } \\
\text { receptor signaling pathway; Innate immune } \\
\text { response-activating signal } \\
\text { transduction;Activation of innate immune } \\
\text { response }\end{array}$ & $8.9 \mathrm{E}-13$ & LUAD \\
\hline Blood coagulation system & $\begin{array}{l}\text { Positive regulation of blood } \\
\text { coagulation;Positive regulation of } \\
\text { hemostasis;Positive regulation of coagulation }\end{array}$ & 6.3 & LUAD \\
\hline $\begin{array}{l}\text { Translation and postranslation } \\
\text { processes }\end{array}$ & $\begin{array}{l}\text { Translation;Peptide biosynthetic process;Amide } \\
\text { biosynthetic process }\end{array}$ & 0.0013 & PRAD \\
\hline
\end{tabular}




\begin{tabular}{|l|l|l|l|}
\hline & $\begin{array}{l}\text { Negative regulation of type B pancreatic cell } \\
\text { development;Superior temporal gyrus } \\
\text { development;Beta-catenin destruction complex } \\
\text { assembly;Regulation of type B pancreatic cell } \\
\text { development;Beta-catenin destruction complex } \\
\text { disassembly }\end{array}$ & 0.041 & PRAD \\
Beta-catenin destruction complex & & \\
\hline
\end{tabular}

\section{References}

1. Ohki, R., Nemoto, J., Murasawa, H., Oda, E., Inazawa, J., Tanaka, N., Taniguchi, T. Reprimo, a new candidate mediator of the p53-mediated cell cycle arrest at the G2 phase.

The Journal of biological chemistry 275(30), 22627-22630 (2000). https://doi.org/10.1074/jbc.C000235200

2. Grzybowska, E. A. Human intronless genes: functional groups, associated diseases, evolution, and mRNA processing in absence of splicing. Biochemical and biophysical research communications $\quad 424(1), \quad 1-6$

(2012). https://doi.org/10.1016/i.bbrc.2012.06.092

3. Liu, X. Y., Fan, Y. C., Gao, S., Zhao, J., Chen, L. Y., Li, F., Wang, K. Methylation of SOX1 and VIM promoters in serum as potential biomarkers for hepatocellular carcinoma. Neoplasma 64(5), 745-753 (2017). https://doi.org/10.4149/neo_2017 513

4. Hung, M. S., Lin, Y. C., Mao, J. H., Kim, I. J., Xu, Z., Yang, C. T., Jablons, D. M., You, L. Functional polymorphism of the CK2alpha intronless gene plays oncogenic roles in lung cancer. PloS one 5(7), e11418 (2010). https://doi.org/10.1371/journal.pone.0011418

5. Xu, W., Li, J. Y. SOX11 expression in mantle cell lymphoma. Leukemia \& lymphoma 51(11), 1962-1967 (2010). https://doi.org/10.3109/10428194.2010.514968

6. Bujko, M., Kober, P., Mikula, M., Ligaj, M., Ostrowski, J., Siedlecki, J. A. Expression changes of cell-cell adhesion-related genes in colorectal tumors. Oncology letters 9(6), 2463-2470 (2015). https://doi.org/10.3892/ol.2015.3107

7. Amigo, J. D., Opazo, J. C., Jorquera, R., Wichmann, I. A., Garcia-Bloj, B. A., Alarcon, M. A., Owen, G. I., Corvalán, A. H. The Reprimo Gene Family: A Novel Gene Lineage in Gastric Cancer with Tumor Suppressive Properties. International journal of molecular sciences 19(7), 1862 (2018). https://doi.org/10.3390/ijms19071862

8. Aviña-Padilla, K., Ramírez-Rafael, J. A., Herrera-Oropeza, G. E., Muley, V. Y., Valdivia, D. I., Díaz-Valenzuela, E., García-García, A., Varela-Echavarría, A., Hernández-Rosales, M. Evolutionary Perspective and Expression Analysis of Intronless Genes Highlight the Conservation of Their Regulatory Role. Frontiers in genetics 12, 654256 (2021). https://doi.org/10.3389/fgene.2021.654256 
9. Wang, Y. Y., Yu, Z., Yi, S. H., Li, Z. J., Li, C. H., Xie, Z. Q., Li, F., Zang, M. R., Hao, M., Qiu, L. G. Expression of SOX11 mRNA in mantle cell lymphoma and its clinical significance 33(7), 556-560 (2012).

10. Yao, Z., Sun, B., Hong, Q., Yan, J., Mu, D., Li, J., Sheng, H., Guo, H. The role of tumor suppressor gene SOX11 in prostate cancer. Tumour biology: the journal of the International Society for Oncodevelopmental Biology and Medicine 36(8), 6133-6138 (2015). https://doi.org/10.1007/s13277-015-3296-3

11. Sander, B., Quintanilla-Martinez, L., Ott, G., Xerri, L., Kuzu, I., Chan, J. K., Swerdlow, S. H., Campo, E. Mantle cell lymphoma--a spectrum from indolent to aggressive disease. Virchows Archiv : an international journal of pathology 468(3), 245-257 (2016). https://doi.org/10.1007/s00428-015-1840-6

12. Croci, G. A., Hoster, E., Beà, S., Clot, G., Enjuanes, A., Scott, D. W., Cabeçadas, J., Veloza, L., Campo, E., Clasen-Linde, E., Goswami, R. S., Helgeland, L., Pileri, S., Rymkiewicz, G., Reinke, S., Dreyling, M., Klapper, W. Reproducibility of histologic prognostic parameters for mantle cell lymphoma: cytology, Ki67, p53 and SOX11. Virchows Archiv : an international journal of pathology 477(2), 259-267 (2020). https://doi.org/10.1007/s00428-020-02750-7

13. Sander B. Mantle cell lymphoma: recent insights into pathogenesis, clinical variability, and new diagnostic markers. Seminars in diagnostic pathology 28(3), 245-255 (2011). https://doi.org/10.1053/j.semdp.2011.02.010

14. Inamdar, A. A., Goy, A., Ayoub, N. M., Attia, C., Oton, L., Taruvai, V., Costales, M., Lin, Y. T., Pecora, A., Suh, K. S. Mantle cell lymphoma in the era of precision medicine-diagnosis, biomarkers and therapeutic agents. Oncotarget 7(30), 48692-48731 (2016). https://doi.org/10.18632/oncotarget.8961

15. Dai, J., Sultan, S., Taylor, S. S., Higgins, J. M. The kinase haspin is required for mitotic histone H3 Thr 3 phosphorylation and normal metaphase chromosome alignment. Genes \& development 19(4), 472-488 (2005). https://doi.org/10.1101/gad.1267105

16. Wang, F., Dai, J., Daum, J. R., Niedzialkowska, E., Banerjee, B., Stukenberg, P. T., Gorbsky, G. J., Higgins, J. M. Histone H3 Thr-3 phosphorylation by Haspin positions Aurora B at centromeres in mitosis. Science 330(6001), 231-235 (2010). https://doi.org/10.1126/science.1189435

17. Jao, T. M., Fang, W. H., Ciou, S. C., Yu, S. L., Hung, Y. L., Weng, W. T., Lin, T. Y., Tsai, M. H., Yang, Y. C. PCDH10 exerts tumor-suppressor functions through modulation of EGFR/AKT axis in colorectal cancer. Cancer letters 499, 290-300 (2021). https://doi.org/10.1016/j.canlet.2020.11.017

18. An, G., Ng, A.Y., Meka, C.S., Luo, G.Z., Bright, S., Cazares, L., Wright, G., Veltri, R; Cloning and Characterization of UROC28, a Novel Gene Overexpressed in Prostate, Breast, and Bladder Cancers. Cancer Research 60 (24), 7014-7020 (2000). 
19. Martin, A. M., Kulski, J. K., Witt, C., Pontarotti, P., Christiansen, F. T. Leukocyte Ig-like receptor complex (LRC) in mice and men. Trends in immunology 23(2), 81-88 (2002). https://doi.org/10.1016/s1471-4906(01)02155-x

20. Denny, J. C., Crawford, D. C., Ritchie, M. D., Bielinski, S. J., Basford, M. A., Bradford, Y., Chai, H. S., Bastarache, L., Zuvich, R., Peissig, P., Carrell, D., Ramirez, A. H., Pathak, J., Wilke, R. A., Rasmussen, L., Wang, X., Pacheco, J. A., Kho, A. N., Hayes, M. G., Weston, N., de Andrade, M. Variants near FOXE1 are associated with hypothyroidism and other thyroid conditions: using electronic medical records for genome- and phenome-wide studies. American journal of human genetics 89(4), 529-542 (2011). https://doi.org/10.1016/j.ajhg.2011.09.008

21. Pan, J., Silva, T. C., Gull, N., Yang, Q., Plummer, J. T., Chen, S., Daigo, K., Hamakubo, T., Gery, S., Ding, L. W., Jiang, Y. Y., Hu, S., Xu, L. Y., Li, E. M., Ding, Y., Klempner, S. J., Gayther, S. A., Berman, B. P., Koeffler, H. P., Lin, D. C. Lineage-Specific Epigenomic and Genomic Activation of Oncogene HNF4A Promotes Gastrointestinal Adenocarcinomas. Cancer research 80(13), 2722-2736 (2020). https://doi.org/10.1158/0008-5472.CAN-20-0390

22. Patrinos, G. P., Forero, D. A. Genome Plasticity in Health and Disease. Elsevier Gezondheidszorg (2020). https://doi.org/10.1016/B978-0-12-817819-5.00009-7

23. Goh, K. I., Cusick, M. E., Valle, D., Childs, B., Vidal, M., Barabási, A. L. The human disease network. Proceedings of the National Academy of Sciences of the United States of America 104(21), 8685-8690 (2007).

24. Huang, S. H., Lo, Y. S., Luo, Y. C., Tseng, Y. Y., \& Yang, J. M. A homologous mapping method for three-dimensional reconstruction of protein networks reveals disease-associated mutations. BMC systems biology 12(Suppl 2), 13 (2018). https://doi.org/10.1186/s12918-018-0537-2

25. Chen, K., Bao, Z., Gong, W., Tang, P., Yoshimura, T., Wang, J. M. Regulation of inflammation by members of the formyl-peptide receptor family. Journal of autoimmunity 85, 64-77 (2017). https://doi.org/10.1016/j.jaut.2017.06.012

26. Le, Y., Murphy, P. M., Wang, J. M. Formyl-peptide receptors revisited. Trends in immunology 23(11), 541-548 (2002). https://doi.org/10.1016/s1471-4906(02)02316-5

27. Liang, W., Chen, K., Gong, W., Yoshimura, T., Le, Y., Wang, Y., Wang, J. M. The Contribution of Chemoattractant GPCRs, Formylpeptide Receptors, to Inflammation and $\begin{array}{llllll}\text { Cancer. Frontiers in endocrinology } & 11, & 17 & \text { (2020). }\end{array}$ https://doi.org/10.3389/fendo.2020.00017

28. Herrera-Oropeza, G. E., Angulo-Rojo, C., Gástelum-López, S. A., Varela-Echavarría, A., Hernández-Rosales, M., Aviña-Padilla, K. Glioblastoma multiforme: a multi-omics analysis of driver genes and tumour heterogeneity. Interface focus 11(4), 20200072 (2021). https://doi.org/10.1098/rsfs.2020.0072 
29. Dai, E., Zhu, Z., Wahed, S. et al. Epigenetic modulation of antitumor immunity for improved cancer immunotheraphy. Molecular Cancer 20, 171 (2021). https://doi.org/10.1186/s12943-021-01464-X

30. Colaprico, A., Silva, T.C., Olsen, C., Garofano, L., Cava, C., Garolini, D., Sabedot, T., Malta, T.M., Pagnotta, S.M., Castiglioni, I., Ceccarelli, M., Bontempi, G., Noushmehr, H. TCGAbiolinks: An R/Bioconductor package for integrative analysis of TCGA data. Nucleic Acids Research (2015). http://doi.org/10.1093/nar/gkv1507.

31. Ramos, M., Schiffer, L., Waldron, L. TCGAutils: TCGA utility functions for data management. R package version 1.14 .0 (2021).

32. Krzywinski, M., Schein, J., Birol, I., Connors, J., Gascoyne, R., Horsman, D., Jones, S. J., Marra, M. A. Circos: an information aesthetic for comparative genomics. Genome research 19(9), 1639-1645 (2009). https://doi.org/10.1101/gr.092759.109

33. Szklarczyk, D., et al. The STRING database in 2021: customizable protein-protein networks, and functional characterization of user-uploaded gene/measurement sets. Nucleic Acid Research 49, D605-D612 (2021).

34. Hagberg, A., Schult, D., Swart, P. Exploring Network Structure, Dynamics, and Function using NetworkX in Proceedings of the 7th Python in Science conference (SciPy 2008), G Varoquaux, T Vaught, J Millman (Eds.), pp. 11-15

35. Lachmann, A., Giorgi, F. M., Lopez, G., Califano, A. ARACNe-AP: gene network reverse engineering through adaptive partitioning inference of mutual information. Bioinformatics 32(14), 2233-2235 (2016). https://doi.org/10.1093/bioinformatics/btw216 